\title{
Significance and interpretation of laboratory tests in pulmonary aspergillosis
}

\author{
MARY P. ENGLISH AND A. H. HENDERSON \\ From the Pathology Laboratory, General Hospital, Bristol
}

SYNOPSIS The significance of the isolation of Aspergillus fumigatus from sputum may be difficult to interpret because of the frequent occurrence of this fungus in the atmosphere. Criteria are given. for classifying positive sputa into two groups, 'fungus scanty' and 'fungus abundant', on the basisoo of cultural and microscopical findings. A relationship between sputum findings thus classified and the occurrence of Aspergillus antibodies in the patient's serum is demonstrated. The diagnostic을 importance of direct microscopical examination of sputum is noted.

The precipitation patterns of sera to the unstandardized antigenic extracts of A.fumigatus ato present available vary greatly. An arbitrary but practical classification of sera is suggested, based on the 'reactivity', or number of precipitation lines produced, and the 'range', or number of antigenic extracts with which the serum reacts.

The examination of sputum for the presence of Aspergillus fumigatus is important in the diagnosis of pulmonary aspergillosis, but the significance of positive cultures is difficult to interpret because the fungus is frequently present in the atmosphere and hence occurs as a mere contaminant of bronchial secretions. The presence in the blood serum of precipitating antibodies to $A$. fumigatus is now also recognized as a valuable diagnostic aid but, because of the present lack of standardized antigenic extracts of the fungus, constant and repeatable results are difficult to obtain.

In the course of a study of pulmonary aspergillosis in chest clinic patients a large number of sputa and sera became available for examination in addition to those received in the normal course of the laboratory routine: this opportunity was taken to assess the validity of laboratory techniques and the usefulness of the findings in clinical diagnoses.

\section{METHODS}

SPUTUM At least two and usually more specimens were examined from each patient, either shortly after collection or following overnight storage at $4^{\circ} \mathrm{C}$. After homogenising by digestion with pancreatin the resulting suspension was centrifuged and the supernatant discarded. Cultures were made by streaking a loopful of sediment thickly on to a plate of glucose-peptone agar containing
$0.05 \mathrm{mg} / \mathrm{ml}$. chloramphenicol and incubating at $37^{\circ} \mathrm{C}$. for $\square$ two to 10 days. Aspergillus spp. were identified by microscopy. Most sputum specimens were also submitted to direct microscopy by macerating a loopful of the sediment in $20 \% \mathrm{KOH}$ on a slide. Prolonged search $\frac{\mathrm{Q}}{\mathrm{Q}}$ at $\times 10$ and $\times 40$ magnifications was often needed before 0 hyphae were found and it was not always possible to $\overrightarrow{\vec{B}}$ distinguish between Aspergillus and Candida hyphae if 3 the latter showed no budding. Only hyphae identified? with certainty as those of Aspergillus were recorded as such.

PRECIPITINS The agar gel double-diffusion method of Ouchterlony (1953) as adapted by Longbottom and 3 . Pepys (1964) was used. To remove the irrelevant $\mathrm{C}-\dot{\mathrm{Q}}$ substance precipitation lines described by Longbottom 3 and Pepys, the discs were soaked in $5 \%$ sodium citrate solution for one hour after the completion of diffusion and before washing (Longbottom, Pepys, and Temple $D$ Clive, 1964, personal communication). The great major-ㅡㅡ. ity of sera, including all positive ones, were screened $\mathrm{N}$ against Bencard's $A$. fumigatus antigens 1 and 2, but a few: at the beginning of the series were tested against three $N$ antigenic extracts kindly supplied by Dr. Longbottom.N In addition, any sera giving weakly positive or doubtful $\omega$ results against these antigens, and negative sera from $<$ patients with positive skin tests or sputum, were retested with a number of antigenic extracts made in this laboratory $\mathbb{\Phi}$ by the method of Longbottom and Pepys (1964). A. fumigatus antibodies were considered to be present if $O$ precipitation lines were obtained with one or more antigens. The sera of some patients were also tested for $\mathbb{Q}$ Candida albicans antibodies using Hollister-Stier Oidio- $\overrightarrow{\mathbb{D}}$ mycin. 


\section{RESULTS}

SPUTUM Sputum from 93 patients was examined: one or more specimens from $27(29 \%)$ of these yielded $A$. fumigatus in culture. On direct microscopy Aspergillus mycelium was found in the sputa of 11 patients from whom the fungus had been isolated but in none of those from whom it had not. Some sputa from patients with aspergilloma were positive on microscopy but negative in culture, but in all other specimens, if the fungus was seen on microscopy it was isolated in culture. In an attempt to overcome the difficulty of assessing the diagnostic value of the isolation of fungus, positive sputa were classified into two groups on the basis of cultural and microscopical results: (1) fungus scanty, when one or two colonies of $\boldsymbol{A}$. fumigatus were cultured from only one specimen and no hyphae found on direct microscopy, (2) fungus abundant, when $A$. fumigatus was cultured from more than one specimen or in large quantity from one specimen and hyphae were found on direct microscopy in many cases.

Table 1 relates sputum findings, classified as above, with the occurrence of precipitins and positive skin tests in the same patients. Sputum findings so classified are of greater diagnostic value to the clinician than when reported simply as 'positive' or 'negative'.

The condition of the mycelium in the sputum was noted in seven of the patients whose sputum was microscopically positive. Most hyphae in sputum from the two patients with aspergilloma were devoid of contents and appeared dead. This would accord with the recognized difficulty of culturing from these cases, and with the fact that the mycelium in a fungus ball is of low viability (Campbell and Clayton, 1964). In contrast, almost all hyphae in sputum from the other five patients contained clearly visible cytoplasm and $\boldsymbol{A}$. fumigatus was easily cultured from these specimens. These five patients included three with allergic aspergillosis, one with invasive aspergillosis confirmed at necropsy, and one with chronic lung disease and positive skin tests and precipitins.

PRECIPITINS Some workers (Stallybrass, 1963: Brönnstam and Hallberg, 1965) consider that crossreactions can occur between antibodies and antigens of Candida and Aspergillus. As C. albicans was present in the sputum of many of our patients and cross-reactions would have invalidated our results, the reactions of the sera of 96 consecutive patients to both antigens were investigated. Cross reactions would be indicated by the existence of precipitation lines to both antigens though only $A$. fumigatus was present in the sputum. Table II shows that in the only two cases in which the double reaction was present $A$. fumigatus and $C$. albicans coexisted in the sputum. Cross-reactions are, therefore, unlikely to have occurred with the antigens used in this study.

\section{TABLE II}

REACTION OF SERA TO A. FUMIGATUS AND C. ALBICANS ANTIGENIC EXTRACTS

$\begin{array}{ll}\text { Positive Reactions to No. of Patients } & \text { Non }\end{array}$

A. fumigatus and C. albicans antigens

A. fumigatus antigens only

C. albicans antigens only

Antigens of neither fungus

$2^{1}$

9

30

Total tested with both antigens

96

${ }^{1}$ Both fungi were present in both these sputa.

It is well recognized that the antigenic extracts available at present are complex and variable and that each yields different precipitation patterns. To compare results on different sera some method of classification is needed. To this end the 'range' of a serum has here been arbitrarily defined as the number of antigenic extracts out of the number tested to which a positive reaction was obtained, and the 'reactivity' as the number of precipitation lines produced against the antigenic extract giving the most lines. This classification is used in Table III. In agreement

TABLE I

RELATIONSHIP BETWEEN SPUTUM FINDINGS AND ANTIBODIES

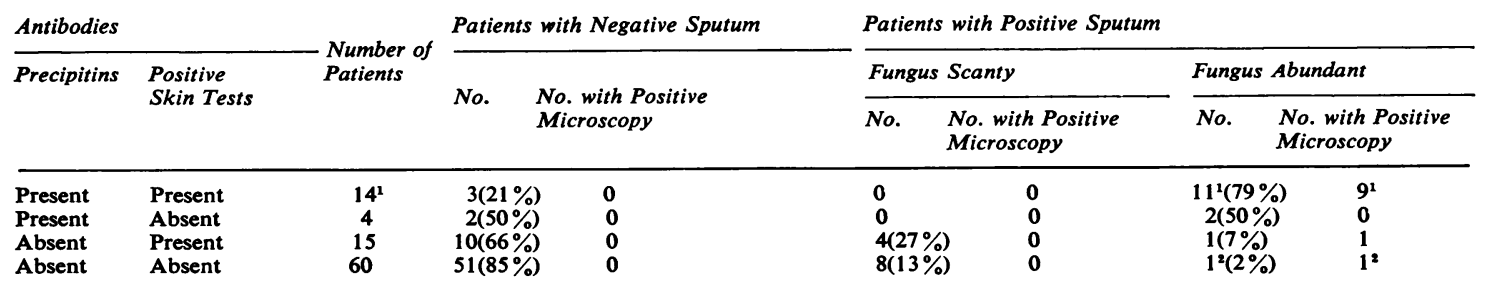

Including nine cases of confirmed aspergillosis of various types.

'Skin test became positive at a later date. 
TABLE III

RANGE AND REACTIVITY OF POSITIVE SERA FROM 21 PATIENTS WITH VARIOUS LUNG CONDITIONS

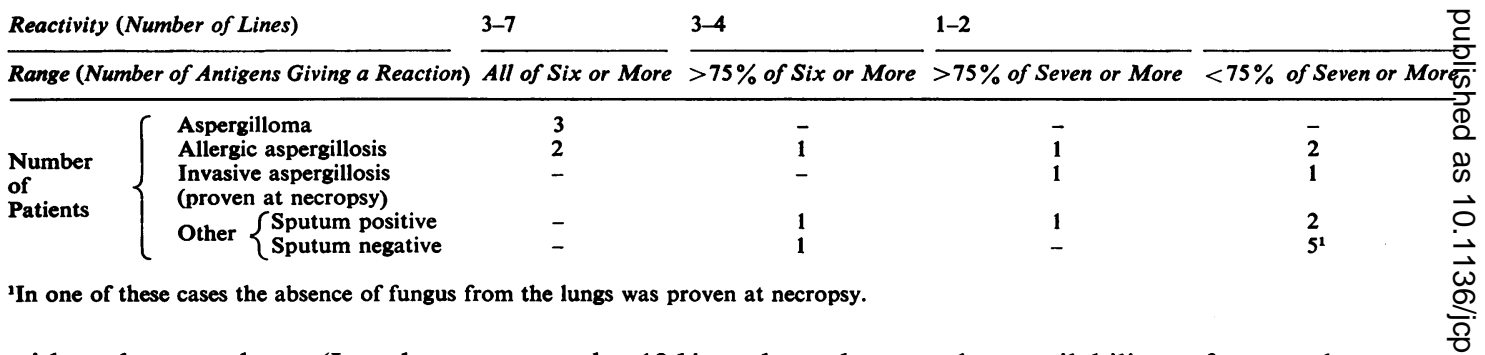

with other workers (Longbottom et al., 1964; Campbell and Clayton, 1964) sera from patients with aspergilloma were found to be among the most reactive and had the widest range, while sera from patients with allergic aspergillosis, though positive in all our cases, varied widely in range and reactivity. Serum from one patient with allergic aspergillosis reacted to only three out of fourteen extracts tried, and that from another to only one out of thirteen. Serum from the two patients with invasive aspergillosis were among those with the smallest range and reactivity, as were sera from five of the six patients in whose sputa no fungus could be demonstrated.

\section{DISCUSSION}

The demonstration of mycelium by direct microscopy in the sputa of 11 out of 15 patients producing abundant fungus in culture and in none of $\mathbf{7 2}$ patients producing scanty fungus or none at all confirms the diagnostic value of this technique, and our experience here differs from that of Orie, de Vries, and Kikstra (1960). The finding of mycelium not only shows that the fungus is growing within the lung and is not merely a contaminant, but its appearance provides evidence of the condition of the organism in the body. However, because of the time-consuming nature of the examination, it might only be practical in the routine laboratory to examine culturally positive sputa. The grouping of patients with positive sputa into those with scanty or abundant fungus adds materially to the clinical usefulness of laboratory results, but its success depends on the availability of several sputumi specimens from each patient.

Longbottom and Pepys (1964) have discussed theio failure of many sera to react with all antigeniw extracts and our results re-emphasize the need to test against a battery of extracts. It is noteworthythat five of our 21 positive sera, including that fromz one case of invasive aspergillosis, failed to react to either of the Bencard antigens, which are the only ones commercially available at present, but gave positive results with some laboratory-made prepara-tions. Our conception of reactivity and range in serao may be useful only until chemically pure antigenic extracts are obtainable. On the other hand, if it is eventually shown that the fungus elicits the produc tion of a large number of recognizable antibodies, the concept may remain useful though the categoriesô will be more precisely defined.

We are greatly indebted to Dr. J. Longbottom for 3 instructing one of us (M.P.E.) in the use of the agar geb precipitation technique, and our thanks are due to $\mathrm{Mr}$ 응 T. J. Morgan and Miss C. A. Walker for technical assis tance. We are grateful to the Medical Research Commit $\bar{\delta}$ tee of the Bristol Royal Hospital for the provision of certain laboratory apparatus.

\section{REFERENCES}

Brönnestam, R., and Hallberg, T. (1965). Acta med. scand., 177, 385. Campbell, M. J., and Clayton, Y. M. (1964). Amer. Rev. resp. Dis., $89,186$.

Longbottom, J. L., and Pepys, J. (1964). J. Path. Bact., 88, 141. ,-- , and Temple Clive, F. (1964). Lancet, 1, 588 .

Orie, N. G. M., de Vries, G. A., and Kikstra, A. (1960). Amer. RevG resp. Dis., 82, 649.

Ouchterlony, O. (1953). Acta path. microbiol. scand., 32, 231. Stallybrass, F. C. (1963). Mycopathologia (Den Haag), 21, 272. 